\title{
Midinfrared photoluminescence of InAsSb quantum dots grown by liquid phase epitaxy
}

\author{
A. Krier, ${ }^{\text {a) }}$ X. L. Huang, and A. Hammiche \\ Department of Physics, Lancaster University, Lancaster LA1 4YB, United Kingdom
}

(Received 27 June 2000; accepted for publication 29 September 2000)

\begin{abstract}
Photoluminescence in the $2-5 \mu \mathrm{m}$ spectral region is reported from $\mathrm{InAs}{ }_{1-x} \mathrm{Sb}_{x}$ quantum dots grown from the liquid phase at $580^{\circ} \mathrm{C}$ on an InAs (100) substrate. Atomic force microscopy shows that coalesced quantum dots and then isolated quantum dots are formed with increasing $\mathrm{Sb}$ composition $(x=0.2-0.3)$ and strain. The $4 \mathrm{~K}$ photoluminescence of the isolated and coalesced quantum dots was observed to peak in the midinfrared at 289 and $316 \mathrm{meV},(4.29$ and $3.92 \mu \mathrm{m})$, respectively. These peaks are due to type II transitions and begin to quench at temperatures above $100 \mathrm{~K}$ as holes become thermally activated out of the quantum dot confinement potential. (C) 2000 American Institute of Physics. [S0003-6951(00)04248-0]
\end{abstract}

InAs and its alloys are promising materials for the fabrication of mid-infrared $(2-5 \mu \mathrm{m})$ optoelectronic devices for use in gas sensors and many other applications. ${ }^{1}$ There is increasing interest in accessing this spectral region using a variety of materials engineering approaches and techniques. In recent years, InSb, GaSb, AlSb, and InAs quantum dots (QDs) have been grown on GaSb and GaAs substrates by molecular beam epitaxy (MBE) and metalorganic vapor phase epitaxy (MOVPE). The resulting photoluminescence emission from these quantum dots was however observed in the near infrared. ${ }^{2-7}$ By comparison there have been no reports of InSb or InAs $\mathrm{As}_{1-x} \mathrm{Sb}_{x}$ quantum dots exhibiting emission in the spectral range $>2 \mu \mathrm{m}$. In this letter we report on the photoluminescence and morphology of $\operatorname{InAs}_{1-x} \mathrm{Sb}_{x}$ quantum dots $(x=0.2-x=0.3)$ encapsulated in InAs and the effect of strain as the $\mathrm{Sb}$ composition was increased laterally along the InAs substrate.

A schematic diagram of the type II $\operatorname{InAs}_{1-x} \mathrm{Sb}_{x} / \mathrm{InAs}$ quantum dot system is shown in Fig. 1. The photoluminescence of InAs is normally dominated by transitions between shallow donors $E_{s}$, deep donors $E_{D}$, and deep acceptors $\left(E_{A_{1}}\right.$ and $E_{A_{2}}$ ). At low temperature, the PL of undoped and $n$-type InAs generally shows two peaks A and B. However, additional peaks $\mathrm{C}$ and $\mathrm{D}$ corresponding to the transitions from $E_{D}$ to $E_{v}$ and from $E_{s}$ to $E_{A_{2}}$, are observed in $p$-type material. ${ }^{8}$ The system affords deep hole confinement $\left(E_{v}\right.$ $\sim 100 \mathrm{meV}$ ) in the quantum dot based on band offset considerations.

The samples for this investigation were grown from the liquid phase using a modified (rapid slider) liquid phase epitaxy (LPE) technique, ${ }^{9}$ which is capable of producing quantum wells and quantum dots. ${ }^{10}$ Under lattice matched growth conditions continuous ultrathin layers (quantum wells) are obtained, but when highly mismatched, quantum dots are produced instead. In the present investigation $\mathrm{InAs}_{1-x} \mathrm{Sb}_{x}$ quantum dots with $x \sim 0.2$ were grown on $n$-type ( $\mathrm{S}$ doped $\left.1 \times 10^{18} \mathrm{~cm}^{-3}\right)$ InAs(100) substrates. Samples of both InAs encapsulated and unencapsulated $\mathrm{InAs}_{1-x} \mathrm{Sb}_{x}$ quantum dots

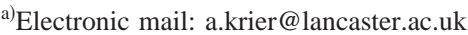

were grown for photoluminescence (PL) and atomic force microscope (AFM) investigation, respectively. The InAs buffer and encapsulation layers were grown from an In-rich melt, whereas the $\mathrm{InAs}_{1-x} \mathrm{Sb}_{x}$ was grown from a pseudobinary melt. The resulting quantum dot layer composition $(x$ $\sim 0.2$ ) was calculated from the $\mathrm{InAs}_{1-x} \mathrm{Sb}_{x}$ phase diagram. ${ }^{11}$ The PL sample was grown at $580^{\circ} \mathrm{C}$ with a ramp cooling rate of $0.3^{\circ} \mathrm{C} / \mathrm{min}$ and using contact times of $300 \mathrm{~s}, 1 \mathrm{~ms}$, and $20 \mathrm{~s}$ for the InAs buffer, InAsSb quantum dot, and InAs capping layers, respectively. All three layers were grown using $25^{\circ} \mathrm{C}$ melt supercooling. The total thickness of the PL sample was $\sim 16 \mu \mathrm{m}$ and the cap layer thickness was $\sim 1 \mu \mathrm{m}$. The corresponding sample for AFM investigation was grown using similar conditions, but without the encapsulation layer. A Topometrix TMX 2000 Explorer AFM was used to investigate the resulting morphology and to obtain the fundamental parameters of the InAs ${ }_{1-x} \mathrm{Sb}_{x}$ quantum dots at different positions (along the passing direction of the melt)

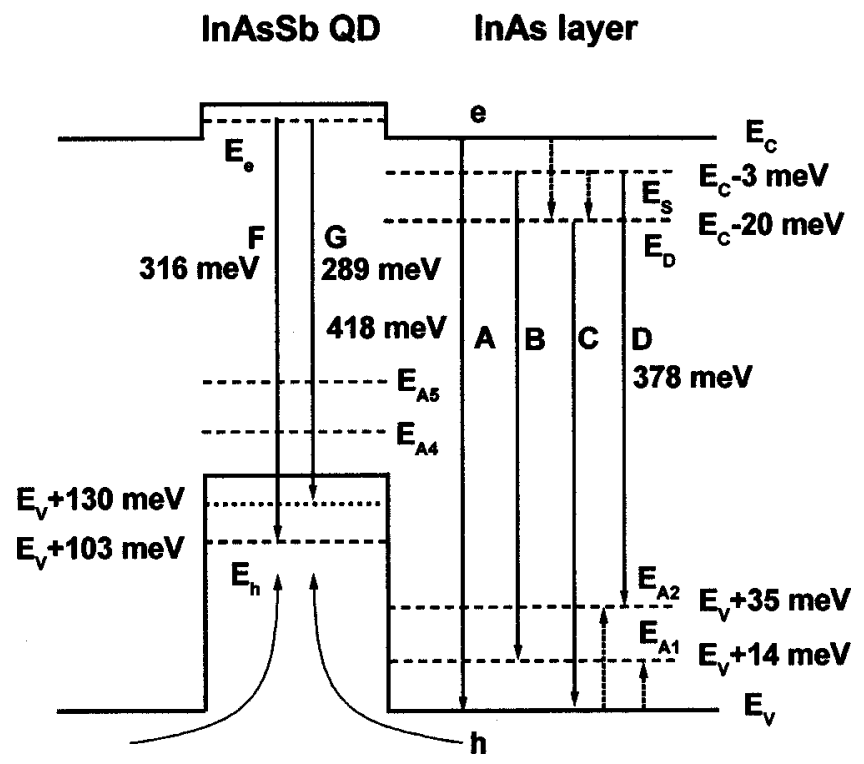

FIG. 1. Schematic diagram showing the type II InAs ${ }_{1-x} \mathrm{Sb}_{x} /$ InAs encapsulated quantum dot. (Energy level assignments are based on literature values where available, confined levels are approximate.) 

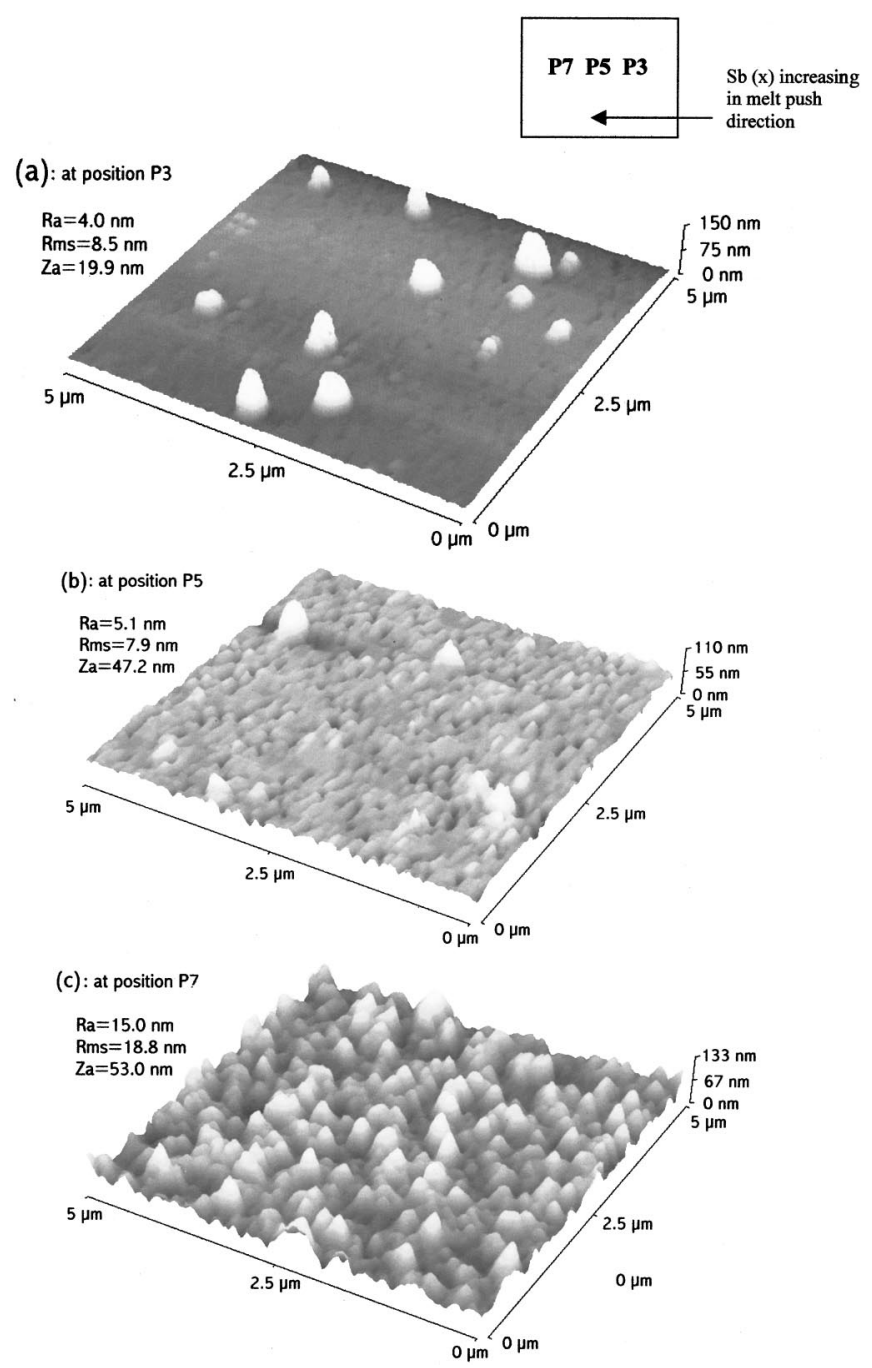

FIG. 2. Atomic force microscope images obtained from unencapsulated $\mathrm{InAs}_{1-x} \mathrm{Sb}_{x}$ quantum dots with increasing $\mathrm{Sb}$ composition grown by LPE and corresponding to the passing direction of the melt across the substrate. Approximate compositions are A $(x=0.20) ; \mathrm{B}(x=0.25)$; and $\mathrm{C}(x$ $=0.30) . \mathrm{R}_{\mathrm{a}}$, rms, and $Z_{a}$ are the area average roughness, the area root-meansquare roughness, and the arithmetic mean height, respectively.

across the substrate. The corresponding low temperature PL spectra were excited using a $5 \mathrm{~W}(514 \mathrm{~nm}$ wavelength) argon-ion laser and detected using a $77 \mathrm{~K} \mathrm{InSb}$ photodiode detector and conventional 4 K PL spectroscopy apparatus for the mid-IR. With this arrangement excitation is indirect, most of the photoexcited carriers are generated within the InAs capping layer and diffuse deeper into the sample where they then recombine within the quantum dots.

Figure 2 shows three AFM images (a), (b), and (c) taken from the sample in regions $P_{3}, P_{5}$, and $P_{7}$ at different positions along the sample as shown in the inset. Even though the contact time is very short $(1 \mathrm{~ms})$, with $25^{\circ} \mathrm{C}$ supercooling there is still local depletion of As from the $\operatorname{InAs}_{1-x} \mathrm{Sb}_{x}$ melt at the growth interface, resulting in a variation in solid composition across the sample along the passing direction of the melt (i.e., Sb increases $P_{3}-P_{7}$ ). This means we are conveniently able to examine the resulting morphology and photoluminescence properties corresponding to different $\mathrm{InAs}_{1-x} \mathrm{Sb}_{x}$ compositions having different amounts of lattice mismatch to the InAs substrate. AFM and PL measurements on an InAs epitaxial control layer grown using the same

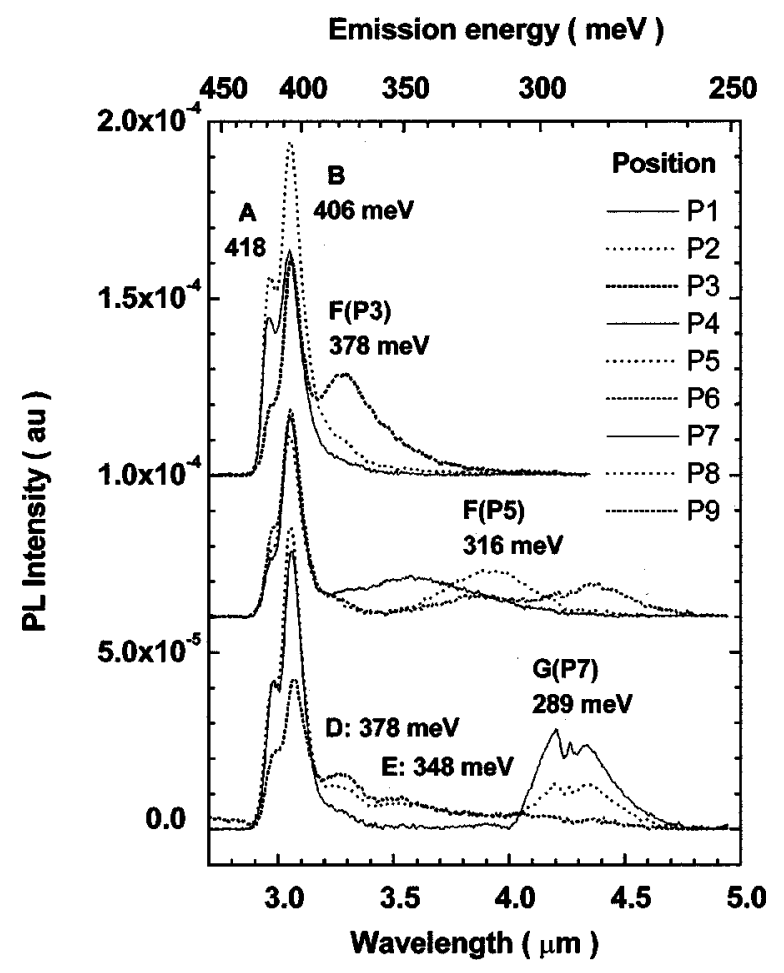

FIG. 3. Photoluminescence spectra measured at $4 \mathrm{~K}$ from the $\operatorname{InAs}_{1-x} \mathrm{Sb}_{x}$ quantum dots with increasing $\mathrm{Sb}$ content from positions $P_{1}$ to $P_{9}$ along the sample. The peaks labeled A-G correspond to the transitions shown in Fig. 1. (Top curves $P_{1}, P_{2}, P_{3}$; middle curves $P_{4}, P_{5}, P_{6}$; lower curves $P_{7}$, $\left.P_{8}, P_{9}\right)$.

techniques revealed a flat surface without any dots and PL characteristic of near band edge recombination, and only weak emission associated with defects.

Using the above technique, we were able to observe the onset of three-dimensional growth of isolated quantum dots from a coalesced (quantum well) layer as a function of strain. Consequently, the $\operatorname{InAs}_{1-x} \mathrm{Sb}_{x}$ in region $P_{3}$ has a relatively small $\mathrm{Sb}$ content $(x=0.2)$ and associated strain. The AFM image of $P_{3}$ shows quite a smooth continuous $\operatorname{InAs}_{1-x} \mathrm{Sb}_{x}$ layer. Some islands about $300 \mathrm{~nm}$ in diameter and $100 \mathrm{~nm}$ in height are visible. This means that a strained epilayer covers most of the area in the vicinity of $P_{3}$, but some large dots have nucleated to help consume the local strain energy. The image from region $P_{5}(x=0.25)$ shows a relatively uniform self-assembled layer of coalesced quantum dots with a few larger islands. ${ }^{2}$ The coalesced quantum dots are about 150 $\mathrm{nm}$ in diameter and $50 \mathrm{~nm}$ in height, with an area density of $\sim 5 \times 10^{9} \mathrm{~cm}^{-2}$. Region $P_{7}$ has the highest $\mathrm{Sb}$ composition $(x=0.3)$ of the three and the AFM image shows isolated quantum dots, some of which have coalesced together. The area density of the isolated dots is $\sim 2 \times 10^{9} \mathrm{~cm}^{-2}$.

Figure 3 shows the $5 \mathrm{~K}$ PL spectra measured from the sample at several different positions $P_{1}-P_{9}$ along the wafer, again corresponding to increasing $\mathrm{Sb}$ composition (where $P_{3}, P_{5}, P_{7}$ relate approximately to AFM images A, B, C). The PL spectra at $P_{1}$ and $P_{2}$ show two peaks $\mathrm{A}$ and $\mathrm{B}$ located at 418 and $406 \mathrm{meV}$, corresponding to band-band transitions (from $E_{c}$ to $E_{v}$ ) and donor-acceptor transitions $\left(E_{s}-E_{A_{1}}\right)$ in InAs, respectively. These originate from the capping and buffer layers but, in the PL at $P_{3}$, another peak (F) located at $378 \mathrm{meV}$ is observed, which correlates with 


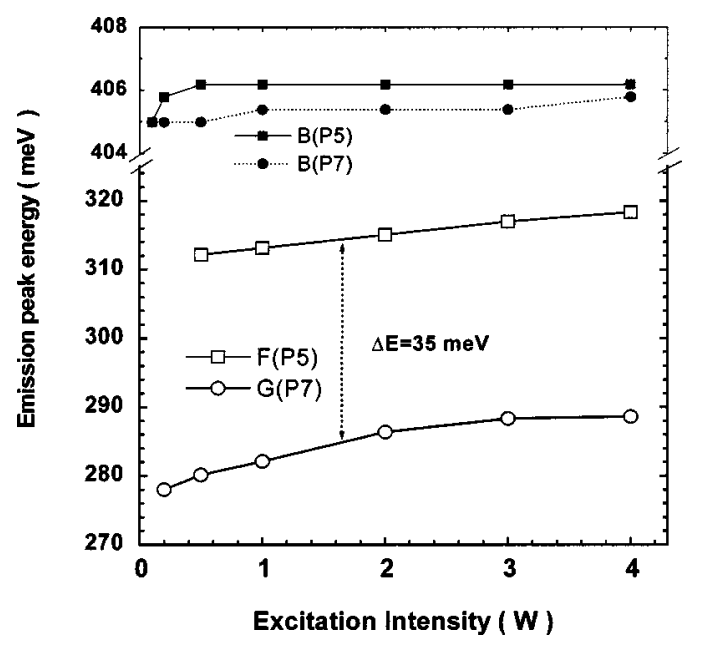

FIG. 4. Variation of emission peak energy with increasing laser power for the principal transitions at $5 \mathrm{~K}$, showing the strong blueshift of the confined state energies; QD, peak $\mathrm{G}\left(P_{7}\right)$, and InAs encapsulation peak $\mathrm{B}\left(P_{7}\right)$.

the $\operatorname{InAs}_{1-x} \mathrm{Sb}_{x}$ " "quantum well', layer (shown in the AFM image at A). At $P_{2}$ the "quantum well" layer is thinner than at $P_{3}$ and peak $\mathrm{F}$ shifts to higher energy and enhances peak B compared with the PL observed at $P_{1}$. In the spectra observed from positions $P_{3}$ to $P_{7}$, peak $\mathrm{F}$ shifts from 378 to $316 \mathrm{meV}$, and decreases in intensity as the $289 \mathrm{meV}$ peak $\mathrm{G}$ increases in intensity. This suggests that as we move across the sample with increasing Sb composition $(x)$ and hence strain, the emission is initially representative of a strained quantum well layer and gradually becomes characteristic of self-assembled coalesced QDs. Then finally at some critical composition $(x \sim 0.25)$ PL is observed mainly from isolated QDs of fixed size at $P_{7} \cdot{ }^{12}$ The two dips in the broad peak $\mathrm{G}$ at around $290 \mathrm{meV}(4.3 \mu \mathrm{m})$ are due to $\mathrm{CO}_{2}$ absorption in the laboratory atmosphere. Moving further along from position $P_{7}$ to $P_{9}$, peak $\mathrm{G}$ decreases in intensity, while the defect related peak D (transitions from $E_{s}$ to $E_{A_{2}}$ ) and its phonon replica peak $E$, as well as peak $\mathrm{B}$ increase in intensity. This is consistent with the overgrowth of the isolated QDs resulting in the production of more deep states $\left(E_{A_{1}}\right.$ and $\left.E_{A_{2}}\right)$ in the InAs in the immediate vicinity outside of the dots which enhances peaks $\mathrm{B}$ and $\mathrm{D}$, as well as introducing recombination centers (perhaps at $E_{A_{4}}$ and $E_{A_{5}}$ ) within the quantum dot to quench peak $\mathrm{G}$.

Figure 4 shows the dependence of emission peak energy on the laser power for the various PL peaks discussed in Fig. 3 above. The peak F blueshifts from 312.2 to $318.4 \mathrm{meV}$ as the excitation intensity increases which is consistent with a type II band alignment for the coalesced QDs. ${ }^{5}$ The blueshift of the isolated quantum dots (peak $\mathrm{G}$ ) with increasing excitation energy is even larger than that of peak F, consistent with a smaller density of states in the isolated QDs compared with the coalesced QDs. The InAs related peaks $\mathrm{B}\left(P_{5}\right)$ and $\mathrm{B}\left(P_{7}\right)$ exhibit only a small blueshift $(1.2 \mathrm{meV})$ by compari- son, consistent with the band-gap recombination. The peak energy of peak $\mathrm{G}$ is about $35 \mathrm{meV}$ lower than that of peak $\mathrm{F}$, because the confinement in the 3D isolated QDs is stronger than the $2 \mathrm{D}$ confinement in the coalesced dots. The integrated intensity of peak $\mathrm{G}\left(P_{7}\right)$ also increases almost linearly with excitation intensity up to $4 \mathrm{~W}$, whereas the intensity of the InAs related peak shows a strong saturation above $2 \mathrm{~W}$.

The temperature dependence of the integrated intensities of these PL peaks provides additional evidence of the strong quantum confinement inside the quantum dots. The PL intensity of the isolated quantum dots, $\mathrm{G}\left(P_{7}\right)$ increases slightly from 5 to $80 \mathrm{~K}$ before rapidly quenching at a temperature above $100 \mathrm{~K}$ with an activation energy of $89 \mathrm{meV}$. The quenching is associated with holes being thermally excited out of the quantum dot confinement potential shown schematically in Fig. 1. By comparison the integrated intensity of the InAs-related peak $\mathrm{B}\left(P_{7}\right)$ quenches rapidly with increasing temperature above $5 \mathrm{~K}$, with an activation energy of only $4.6 \mathrm{meV}$.

Mid-infrared photoluminescence from $\operatorname{InAs}_{1-x} \mathrm{Sb}_{x}$ quantum dots grown by LPE has been observed at $4.3 \mu \mathrm{m}$. The dependence of the resulting PL with increasing Sb composition and strain revealed distinct peaks at 316 and $289 \mathrm{meV}$ which resulted from type II transitions originating from coalesced and isolated dots, respectively. The PL emission persisted up to $210 \mathrm{~K}$ but began to quench rapidly above $100 \mathrm{~K}$ with an activation energy which corresponds approximately to the hole confinement energy in the $\mathrm{InAs}_{1-x} \mathrm{Sb}_{x}$ quantum dot.

The authors wish to thank EPSRC for the award of a research grant to support this work.

\footnotetext{
${ }^{1}$ See, for example, B. Matveev, N. Zotova, S. Karandashov, M. Remennyi, N. Il'inskaya, N. Stus', V. Shustov, G. Talalakin, and J. Malinen, IEE Proc.: Optoelectron. 145, 245 (1998), Special Issue on Mid-IR devices and materials and papers therein (see also Vol. 144 for additional related papers).

${ }^{2}$ M. Yano, Y. Seki, H. Ohkawa, K. Koike, S. Sasa, and M. Inoue, Jpn. J. Appl. Phys., Part 1 37, 2455 (1998).

${ }^{3}$ B. R. Bennett, P. M. Thibado, M. E. Twigg, E. R. Glaser, R. Magno, B. V. Shanabrook, and L. J. Whitman, J. Vac. Sci. Technol. B 14, 2195 (1996).

${ }^{4}$ B. R. Bennett, R. Magno, and B. V. Shanabrook, Appl. Phys. Lett. 68, 505 (1996).

${ }^{5}$ E. R. Glaser, B. R. Bennett, B. V. Shanabrook, and R. Magno, Appl. Phys. Lett. 68, 3614 (1996).

${ }^{6}$ S. P. Guo, H. Ohno, A. D. Shen, Y. Ohno, and F. Matsukura, Jpn. J. Appl. Phys., Part 1 37, 1527 (1998).

${ }^{7}$ E. Alphandery, R. J. Nicholas, N. J. Mason, B. Zhang, P. Mock, and G. R. Booker, Appl. Phys. Lett. 74, 2041 (1999).

${ }^{8}$ H. H. Gao, A. Krier, and V. V. Sherstnev, Semicond. Sci. Technol. 14, 441 (1999).

${ }^{9}$ A. Krier, Z. Labadi, and J. Richardson, IEE Proc.: Optoelectron. 145, 297 (1998).

${ }^{10}$ A. Krier, Z. Labadi, and A. Hammiche, J. Phys. D 32, 1 (1999).

${ }^{11}$ G. B. Stringfellow and P. E. Greene, J. Phys. Chem. Solids 30, 1779 (1969).

${ }^{12}$ Semiconductors and Semimetals, edited by M. Sugawara (Academic, New
} York 1999), Vol. 60, pp. 168-172. 